\section{Facharztprüfung zur Erlangung des Schwerpunktes Pädiatrische Radiologie zum Facharzttitel FMH für Radiologie}

Entsprechend dem neuen Weiterbildungsprogramm vom 1.1.2001 können dieses Jahr erstmals zum Facharzttitel FMH für Radiologie Prüfungen zur Erlangung des Schwerpunktes Pädiatrische Radiologie abgelegt werden.
Datum: Samstag, 1. September 2001

Ort: Hôpital cantonal universitaire, Genève

Prüfungsgebühr: Die Prüfungsgebühr beträgt für Mitglieder der SGR/SGPR Fr. 300.-, für Nichtmitglieder Fr. 450.-.

Auskunft: PD Dr. med. Heinz Tschäppeler, Inselspital, Röntgenabteilung Kinderklinik, 3010 Bern, Tel. 03163295 01, E-mail heinz.tschaeppeler@insel.ch.

Anmeldung: Sekretariat PD Dr. med. St. Duewell, Frau B. Haldner, Kantonsspital Frauenfeld, Radiologie, 8500 Frauenfeld, Tel. 05272371 63, Fax 05272373 48, E-mail bernadette.haldner@kttg.ch.

Zulassungsbedingungen: Bestandene 2. Teilprüfung Radiologie

Anmeldeschluss: 31. Mai 2001
Examen de spécialiste pour l'obtention de la formation approfondie en radiologie pédiatrique à adjoindre au titre de spécialiste FMH en radiologie

Selon le nouveau programme de formation postgraduée du $1^{\text {er }}$ janvier 2001, un examen pourra être passé pour la première fois cette année attestant d'une formation approfondie en radiologie pédiatrique à adjoindre au titre de spécialiste FMH en radiologie.
Date: Samedi, $1^{\text {er }}$ septembre 2001

Lieu: Hôpital cantonal universitaire, Genève

Frais d'inscription: Montant de la taxe d'examen pour membre de la SSR/SSRP Fr. 300.-, pour nonmembre Fr. 450.-.

Renseignements: Dr Heinz Tschäppeler, PD, Hôpital de l'lle, Service de radiologie de la clinique pédiatrique, 3010 Berne, tél. 03163295 01, e-mail heinz.tschaeppeler@insel.ch.

Inscription: Secrétariat du Dr St. Duewell, PD, Mme B. Haldner, Hôpital cantonal de Frauenfeld, Radiologie, 8500 Frauenfeld, tél. 05272371 63, fax 05272373 48, e-mail bernadette.haldner@kttg.ch.

Conditions d'inscription: la réussite de la $2^{\text {ème }}$ partie de l'examen de spécialiste en radiologie.

Délai d'inscription: 31 mai 2001 